\title{
Magnetic Shield Effectiveness in Low Frequency
}

\author{
A. Keshtkar, A. Maghoul and A. Kalantarnia
}

\begin{abstract}
Since the early days of radio and telegraph communications, it has been known that a spark gap generates electromagnetic waves rich in spectral content (frequency components) and that these waves can cause interference or noise in various electronic and electrical devices such as radio receivers and telephone communications. Electromagnetic pulses are fields of energy that they can damage electrical and electronic circuits at once. New technology of microelectronic circuits is sensitive to interference power. An intentional or inadvertent risk and unforeseen create irreparable damages, so that it is important to investigate electromagnetic interference from viewpoint: generation source, the effectiveness on electronic components and safekeeping methods. One of the prevalent methods for safekeeping against these pulses is shielding. In this paper, at first, the effect of magnetic field intensity is investigated in frequency selected $(0-545 \mathrm{~Hz})$ for various shields such as: paramagnetic materials, diamagnetic materials and ferromagnetic materials and shielding effectiveness is calculated for various low frequencies in one point in shield and shielding effectiveness variation with increase in frequency for any material is obtained. In The following of paper, the effect of magnetic field intensity is investigated on shield's two apertures and six apertures. Then, the apertures are displaced on shield surface and shielding effectiveness variation is obtained. This apertures displacement has done for various shield materials. Finally, the effects of composite shields are studied to shielding effectiveness. These shields have combined to two materials. We obtained interesting results from composite shields. In this paper, solution method has chosen 2DFEM and the whole of simulations have done Cosmos/M.
\end{abstract}

Index Terms - Shielding effectiveness, Helmholtz coil, paramagnetic material, diamagnetic material, ferromagnetic material, low frequency.

\section{INTRODUCTION}

With the increase applications of electronic equipment, the electromagnetic interfering problem becomes more and more serious. Also, increasing use of electrical power, high magnetic fields of power frequency are often encountered in our environment. These high field strengths may cause disturbances on video display units. The interference occurs mainly as frame disturbances when the magnetic flux density is above $0.5-1 \mu \mathrm{T}[1]$. One of ways to limit the penetration electromagnetic fields into systems is shielding. Electromagnetic shielding is the process of limiting the penetration of electromagnetic fields into a space, by

Manuscript received March 11, 2011; revised July 11, 2011.

A. Keshtkar is with the Faculty of Engineering and Technology, Imam Khomeini International University, Ghazvin (akeshtkar@gmail.com).

A. Maghoul is currently a researcher with EMC department in space research center of Iran, Tehran, Iran. (amir.maghoul@yahoo.com).

A. Kalantarnia is currently a Faculty member of Applied and Science Technology, Hamedan, Iran (ali.kalantarnia@gmail.com). blocking them with a barrier made of conductive material. Typically it is applied to enclosures, separating electrical devices from the 'outside world', and to cables, separating wires from the environment the cable runs through. The shape of the shield is in many cases restricted by practical limitations, and only partial shielding may be possible to use. In electromagnetic, shielding effectiveness (SE) is a concise parameter generally applied to quantify shielding performance [2]? The shielding effectiveness (SE) is equal to ratio quantities at the receptor without the enclosure and with enclosure:

$$
\begin{aligned}
& \text { for } \mathrm{E} \text { - field: } \quad \mathrm{SE}_{\mathrm{dB}}=20 \log \left(\frac{\mathrm{E}_{0}}{\mathrm{E}_{s}}\right) \\
& \text { for } \mathrm{H} \text { - field: } \quad \mathrm{SE}_{\mathrm{dB}}=20 \log \left(\frac{\mathrm{H}_{0}}{\mathrm{H}_{s}}\right)
\end{aligned}
$$

where, $\mathrm{E}_{0}$ and $\mathrm{H}_{0}$ represent quantities at the receptor without shielding body, $\mathrm{E}_{\mathrm{s}}$ and $\mathrm{H}_{\mathrm{s}}$ denote quantities at the receptor with a shielding barrier between the emitter and receptor. Owing to some special demands, the lower frequency need shielding is $50 \mathrm{~Hz}$ or even $0.01 \mathrm{~Hz}$ sometimes. This special shielding is used for the protection against the electromagnetic radiation field generated by generator or large-scale voltage transformer, the experiment of measuring and regulating, and the laboratory of the electron microscope test [3].

The shielding of low frequency, low Impedance and high intensity electromagnetic waves is a complex task. Indeed, as is known from the Schekunoff's theory [4]. Reflection losses are significantly reduced for low impedance waves, i.e. when the wave impedance value is close to that of the intrinsic impedance of the shield, and absorption losses diminish for low frequency operation. The use of materials that exhibit a magnetic structure with not vanishing macroscopic magnetic moment, i.e. ferromagnetic bodies, usually increases the shielding of magnetic fields. Essentially, this is because of the shielding of the result of two synchronously occurring physical mechanism: the flux shunting through the high permeability of the materials and redirection of flux due to induced eddy current [5].

In this paper, the effect of magnetic field intensity is studied in low frequency for shields with various materials. Also, the effect of magnetic field intensity is investigated on shield with aperture, by frequency variation and could see the diversion of field lines on shield. Then, the effect of increase in apertures number on shielding effectiveness has obtained. In following paper, the apertures are displaced on shield surface and shielding effectiveness variation is obtained. Finally, the effects of composite shields were investigated to shielding effectiveness. Also, it is necessary to know, in this paper, shielding effectiveness in magnetic field is investigated. 


\section{Electromagnetic Compatibility Test AND SHIElD ENCLOSURE}

At low frequencies, we can regard the magnetic field as a result of the electric current flow and the magnetization of surrounding materials. There are two basic methods for shielding against low-frequency magnetic sources: diversion of the magnetic flux with high-permeability materials and the generation of opposing flux via Faraday's law, commonly known as the "shorted turn method." The diversion of magnetic flux with a low-reluctance (high permeability) path is necessary to design. In generally, there are two factors that may influence shielding effectiveness in low frequencies [6]:

1 . The permeability of ferromagnetic materials decreases with increasing frequency.

2. The permeability of ferromagnetic materials decreases with increasing magnetic field strength.

It is necessary to generate a field of known uniformity for shielding test and electromagnetic compatibility (EMC) test. There are some ways for uniform field generation. Among the rest can mention to below cases:

1. Solenoid

2. Toroid

3. Spherical coil with variable winding

4. Helmholtz coil

Helmholtz coil in the above cases, have the most of applications in EMC tests. Helmholtz coil is constructed with either circular or square coils. The field generates is the sum of the fields generated by the two spaced coils [7].

\section{Simulation StRUCtURE}

At low frequencies, the magnetic field is due either to the electric current flowing in conductors of various geometries or to the magnetization of surrounding ferromagnetic materials. The classical strategy for reducing quasi-static magnetic fields in a specific region consists in inserting a shield of appropriate material, whose properties are used to alter the spatial distribution of the magnetic field emitted by the source. In fact, the shield causes a change in the behavior of the field, diverting the lines of the magnetic induction away from the shielded region. Copper and aluminum are common shield materials in traditional high-frequency shield design, whereas highly permeable metals are used in magneto static problems. Using materials with very high relative permeability, such as mu-metal, results in a costly design when the shield is as room size [8].
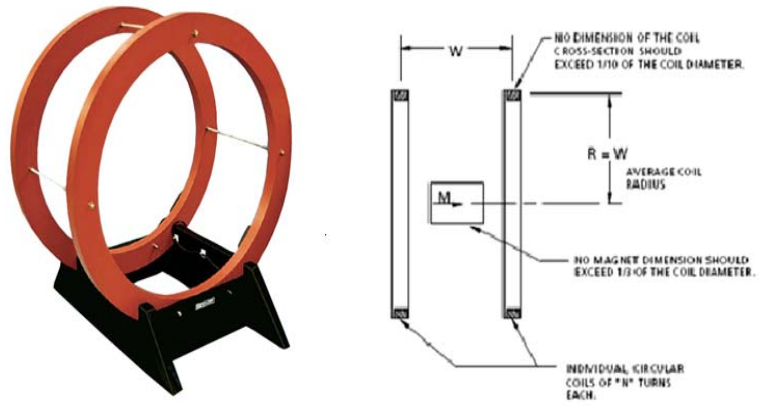

Fig.1. Helmholtz coil Configuration [9]

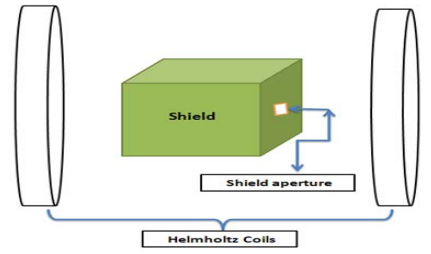

(a)

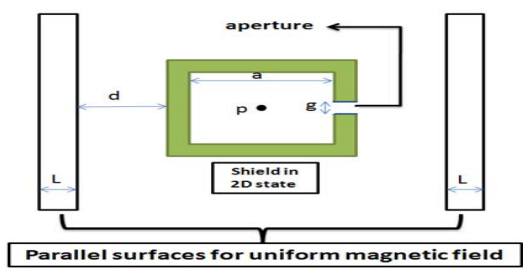

(b)

Fig.2. (a) shield enclosure in coils, (b) approximated structure

At first, the workspace is created to simulate proposed configuration and the proposed structure is demonstrated in 2D state. In above structure, Fig (a) is approximated Fig (b). In proposed structure, Helmholtz coils is approximated to two surfaces because, the aim of them is uniform field creation. Simulated configuration has been shown in Fig.2 with $(\mathrm{L}=0.15 \mathrm{~m}),(\mathrm{d}=1.425 \mathrm{~m}),(\mathrm{a}=0.9 \mathrm{~m})$. The structure is symmetrical. One aperture created in right side on shield surface. The shield dimensional is $1 \mathrm{~m} \times 1 \mathrm{~m}$ and shield thickness also is $5 \mathrm{~cm}$. Also, the current of coil surface is equal $10 \mathrm{~A}$ in the opposite direction. The following simulation, at first, the type of shield chose paramagnetic and diamagnetic materials. Then, the shielding effectiveness has been obtained in point $(\mathrm{P})$ with frequency variation. As it was seen in Fig.3, the shield in middle of coils diverts uniform field lines. Also, field lines penetrate in shield enclosure by aperture. In following, it is shown in fig.4, the shielding effectiveness factor increases by increase in frequency for diamagnetic materials.

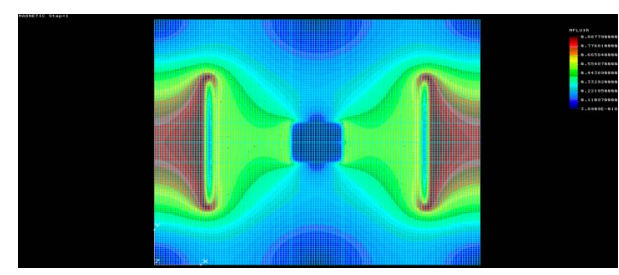

(a)

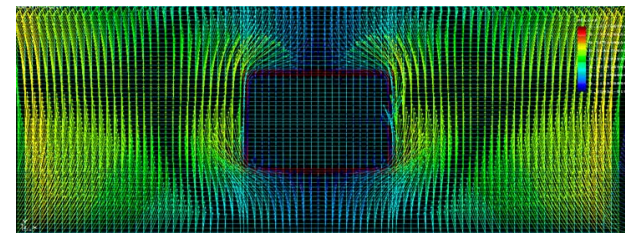

(b)

Fig.3. (a) contour plot, (b) the effect of field lines in shield

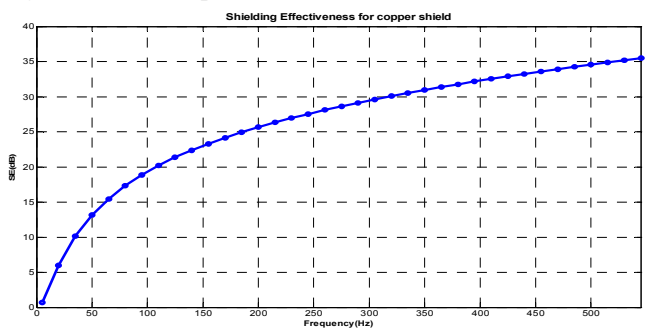

(a) 


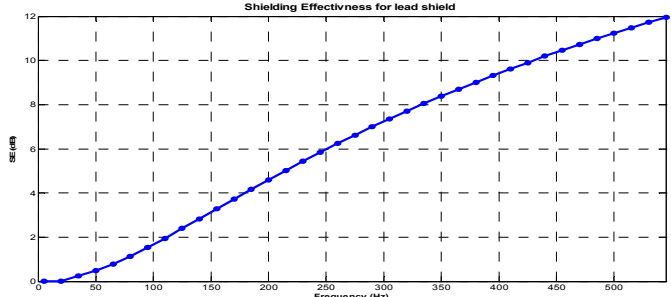

(b)

Fig.4. shielding effectiveness on shield enclosure: (a) Copper, (b) lead

In low frequencies, skin effect for Shield enclosure is large, but the increase of frequency caused to skin effect reduction. Consequently, shielding effectiveness increased the reduction of skin effect.

Now, the type of shield material is selected from high preamble materials as: Permalloy, Nickel and it is assumed to fix their permeability factor by increase in frequency. At first, field intensity is very high and the greater fields penetrate inside of shield, when the frequency is increasing. There for, shielding effectiveness decreases. In following, with increase in frequency, after initial frequencies, penetration field inside of shield reduces. This occurs because of eddy currents create on shield. Eddy currents produce field lines, they arrange in opposite of incident fields. Then, penetration fields decrease in shield. There for, shielding effectiveness increases.

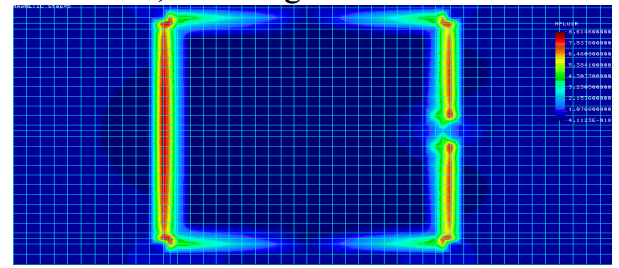

(a)

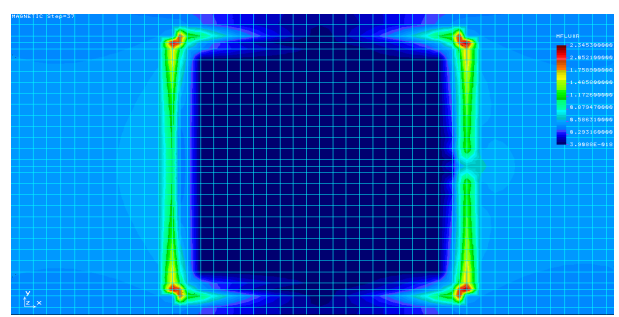

(b)

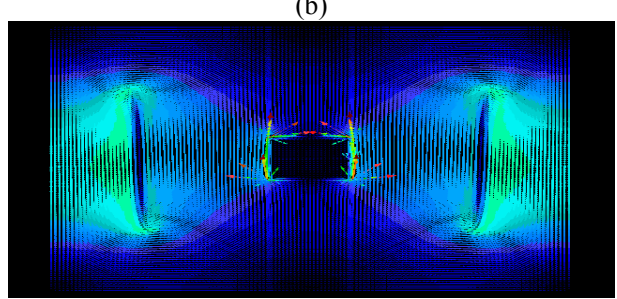

(c)

Fig.5. (a) field effect on shield in below frequencies of $100 \mathrm{~Hz}$, (b) field effect on shield in above frequencies of $100 \mathrm{~Hz}$, (c) field lines on nickel shield.

Fig. 5 shows the effect of field intensity on shield for nickel material. The reason of decrease in field on shield enclosure is eddy currents. As shown, after a little reducing of shielding effectiveness for nickel shield, it increases. The Fig.6 shows shielding effectiveness for nickel shield in low frequencies. For high permeability materials as: Nickel, Iron, after slightly reduction, shielding effectiveness increase with frequency growth and its reason can be justified by shield resonance.

Now, the type of shield material is changed. The shield material selected from Permalloy. It is necessary to remind that permeability parameter for this material is assumed to fix in this paper. As mentioned before, the aim of our research is to investigate the effect of high permeability materials on shielding effectiveness.

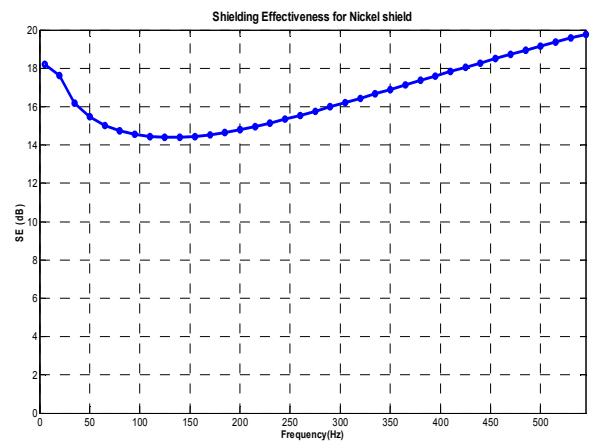

Fig.6. shielding effectiveness on nickel shield

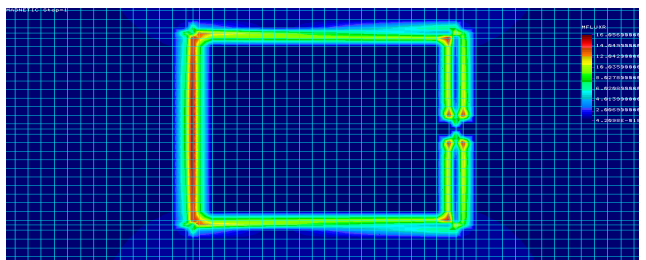

(a) $\mathrm{F}<100 \mathrm{~Hz}$

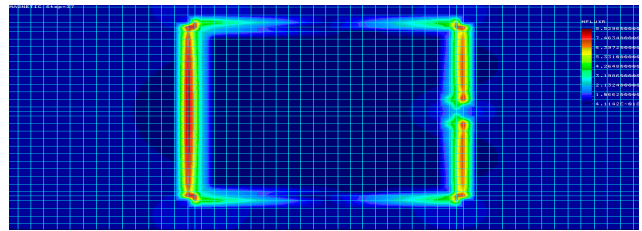

(b) $\mathrm{F}>100 \mathrm{~Hz}$

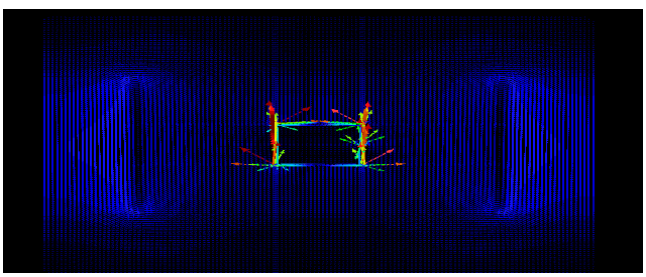

(c)

Fig.7. (a), (b) shielding effectiveness on nickel shield, (c) field lines on permalloy shield

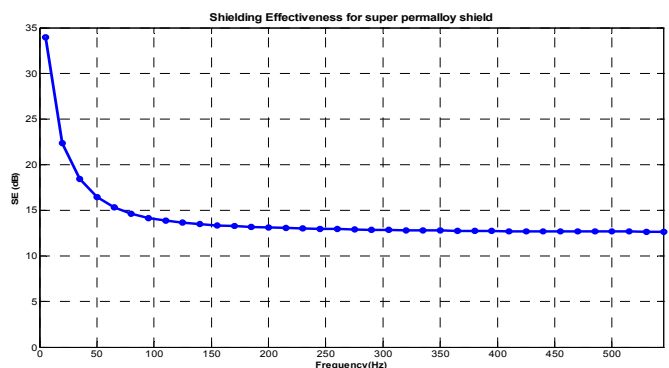

Fig.8. shielding effectiveness on permalloy shield

As shown, shielding effectiveness decreased suddenly in initial frequencies. Skin depth in these materials is really less than shield thickness with increase in frequency and it is reason for decreasing the shielding effectiveness. In following, with increase in frequency, create tradeoff between decreases in skin effect and eddy currents. Eddy currents generate high field intensity on shield surface to improve shielding effectiveness and decrease incident fields and it is caused to fix shielding effectiveness approximately after suddenly reducing in initial frequencies. 


\section{APERTURES ON SHIELD ENClOSURE}

Shielded enclosures are usually employed to protect against radiation from external EM fields and leakage effects from interior components. There are numerous cases where openings in an otherwise contiguous shield cannot be avoided for practical reasons. One of the more common ones is the need to ventilate the internal electronics for thermal reasons. Fans are frequently employed to move hot air inside the shield to the outside.

The efficiency of enclosures is often compromised by apertures and slots located on the walls of the enclosure. Therefore, apertures are impotent factor to determine shielding effectiveness. In fact, the practical shielding effectiveness is not determined by material characteristics but is limited by necessary apertures and discontinuities in the shielding.

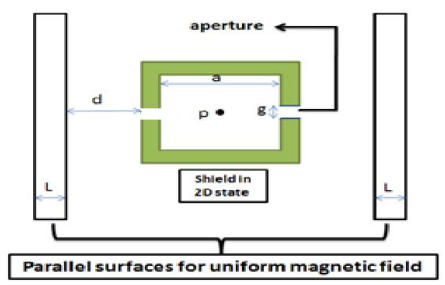

Fig.9. approximated structure with two apertures

In the following of paper, the number of apertures is investigated the shielding effectiveness for different materials. Of course, the apertures selected randomly, the sizes of apertures and the distance of apertures are equal. Approximated model is shown in Fig.9.

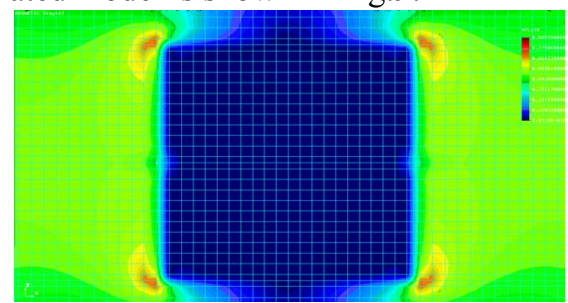

(a)

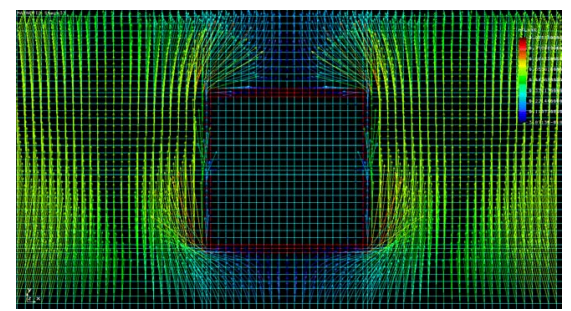

(b)

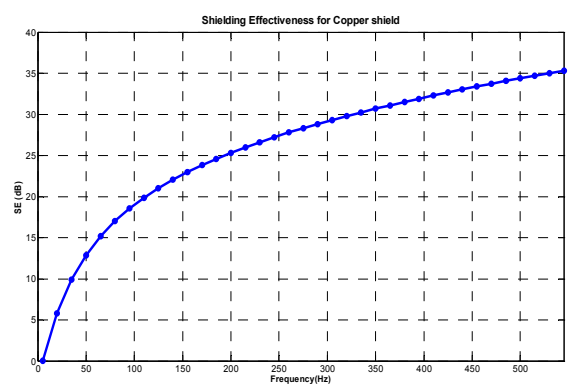

(c)

Fig.10. (a) contour plot, (b) the effect of field lines on copper shield's two apertures, (c) shielding effectiveness on copper shield's two apertures.

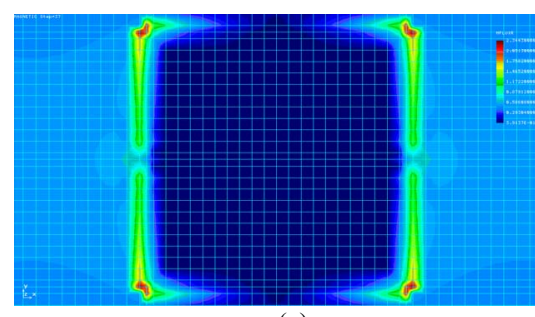

(a)

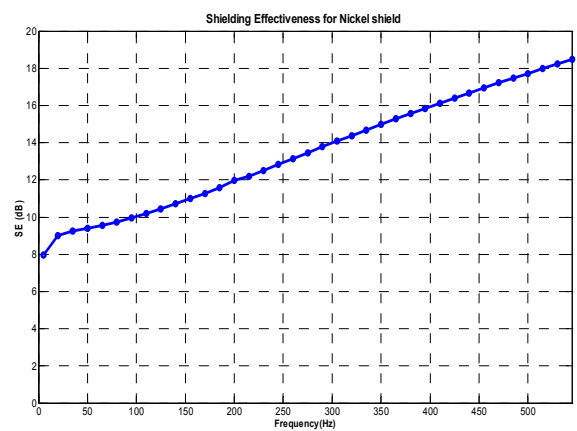

(b)

Fig.11. (a) Contour plot (b) shielding effectiveness on nickel shield's two apertures

At first, copper shield is selected with two apertures, in first stage. Fig.10 shows its results. A lot of fields penetrated in shield enclosure. Consequently, the shielding effectiveness decreased. In following, the apertures are increased to six apertures and investigated field penetration.

Again, shield enclosure is examined with six apertures and shielding effectiveness is obtained. Shield materials are selected from: copper, nickel and Permalloy. Approximated shield is shown in Fig.12. All of apertures are equal and placed face to face in 2D.

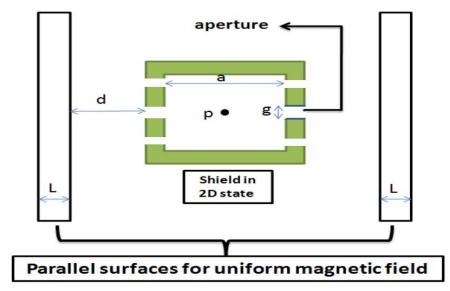

Fig.12. approximated structure with six apertures

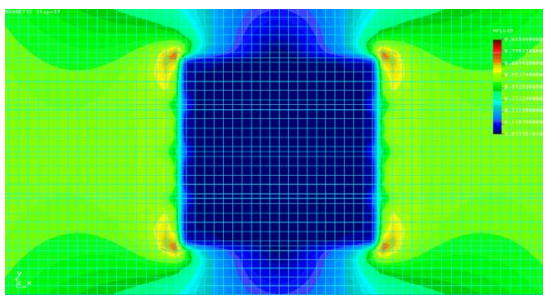

(a)

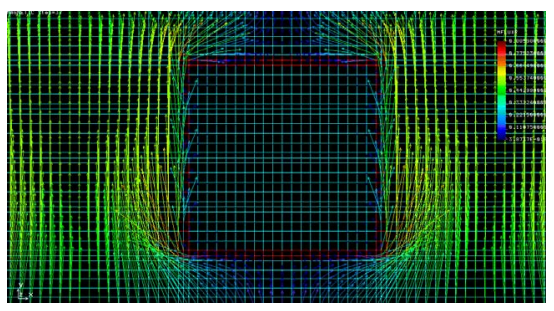

(b)

Fig.13: (a) Contour plot, (b) the effect of field lines on copper shield's six apertures 


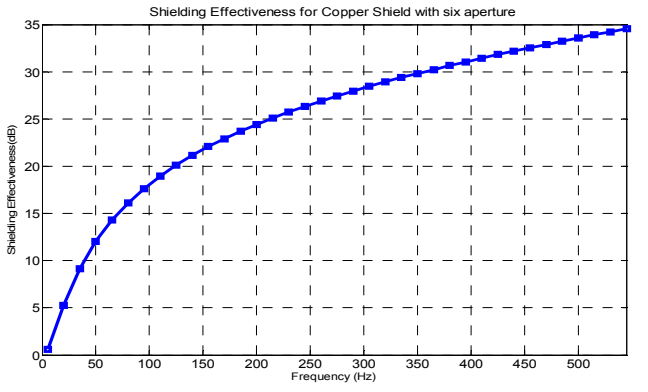

Fig.14: (a) shielding effectiveness on Copper shield's six apertures.

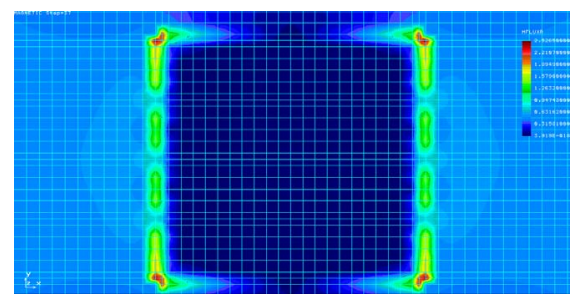

(a)

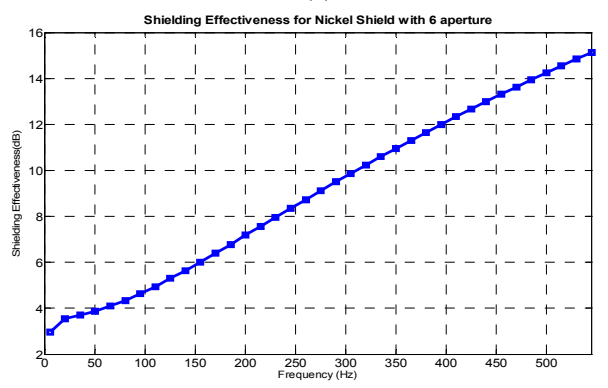

Fig.15: (a) Contour plot, (b) shielding effectiveness on nickel shield's six apertures.

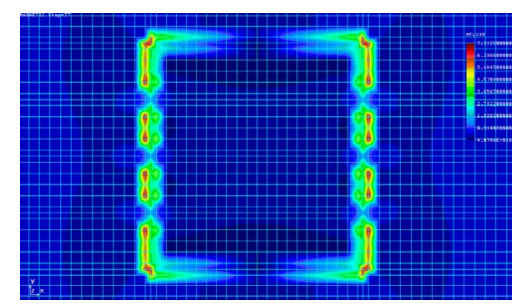

(a)

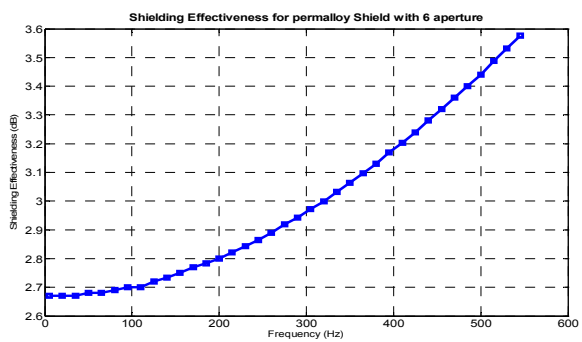

(b)

Fig.16: (a) Contour plot, (b) shielding effectiveness on permalloy shield's six apertures.

As seen, apertures decreased the shielding effectiveness and this reduction for high permeable material is considerable. The above results show, the apertures are important factor to determine shielding effectiveness, also they determine the quality of shield.

Now, aperture position is changed. The goal of this displacement is that, the variation of shielding effectiveness for various materials is considered. Of course, apertures sizes in shield are equal with $\mathbf{g}$ and the material of shield is changed.
The material of shield is composed of: aluminum, nickel, mumetal. Because of symmetry in fig.17, the effect of field intensity on shielding factor is equal for fig.17 (a), fig (b). It is obvious to the shielding effectiveness reduces with shorting the space between point $\mathrm{P}$ and the aperture.

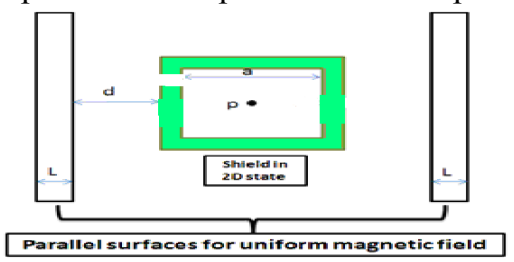

(a)

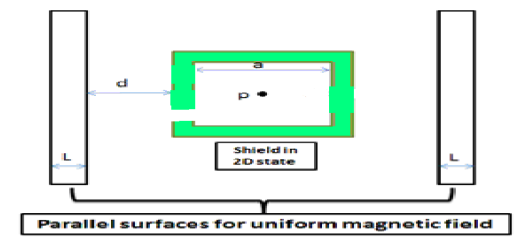

(b)

FiFig.17. approximated structure with aperture displacement, (a) the aperture is left corner of above, (b) the aperture is left

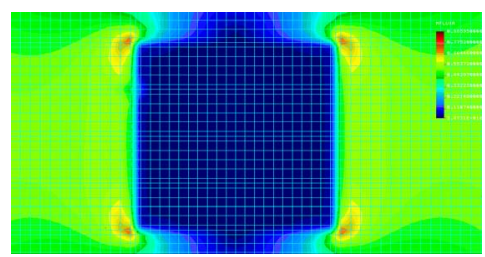

(a)

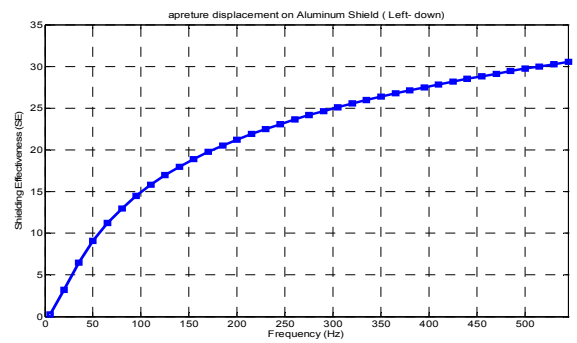

(b)

FiFig.18. (a) Contour plot, (b) shielding effectiveness on aluminum shield with aperture displacement

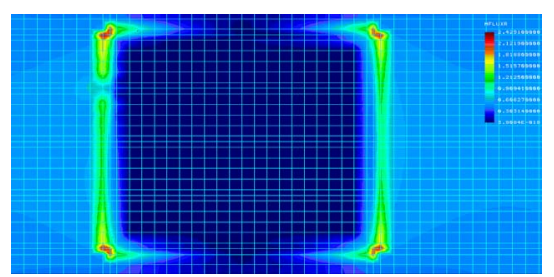

(a)

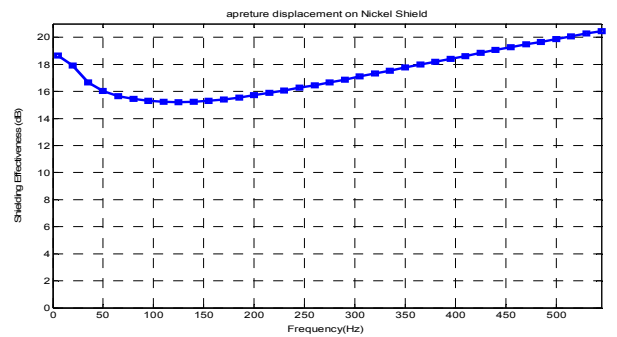

(b)

Fig.19. (a) Contour plot, (b) shielding effectiveness on nickel shield with aperture displacement 


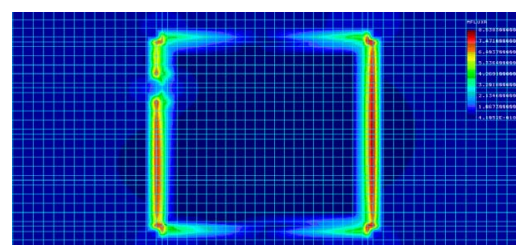

(a)

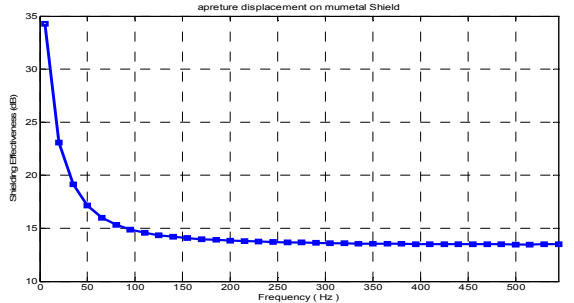

(b)

Fig.20. (a) Contour plot, (b) shielding effectiveness on mumetal shield with aperture displacement

Attention to above results, the aperture placement is important factor to determine shielding effectiveness. Therefore, if the aperture distance be closer to measuring Fig.24. (a) Contour plot, (b) shielding effectiveness on aluminum- nickel shield point, then a lot of fields penetrate in shield and shielding effectiveness decreases.

\section{THE COMPOSITION OF MATERIALS FOR SHIELD DESIGNING}

In last section, the composite shield is investigated to have six apertures on shield. Shield material is composed of composite material as: Nickel-aluminum, Mumetalaluminum, and Nickel-Mumetal. They have connected together as Fig.21 in 2D. As we know, shielding factor depends on shield configuration severely. The shield configuration is an important parameter to cause decrease or increase in shielding effectiveness.

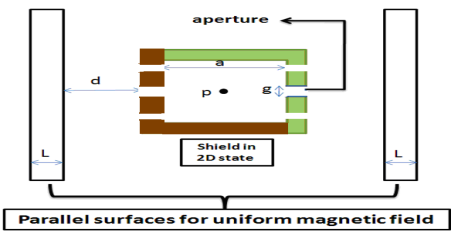

Fig.21. approximated structure with six apertures for composite shield

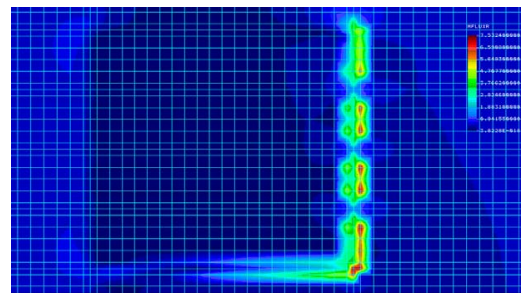

(a)

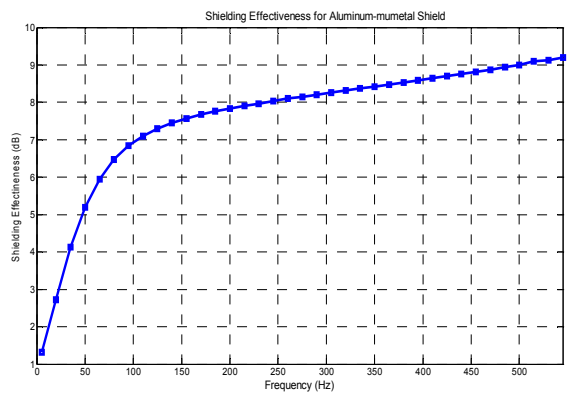

(b)

Fig.22. (a) Contour plot, (b) shielding effectiveness on aluminum- mumetal shield

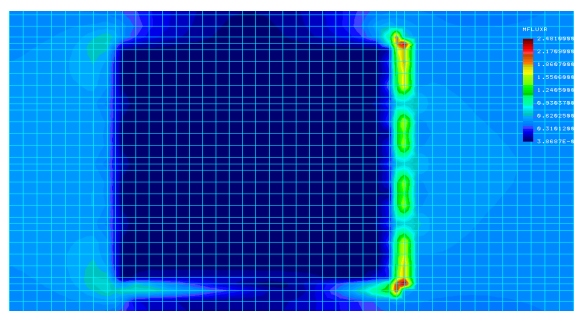

(a)

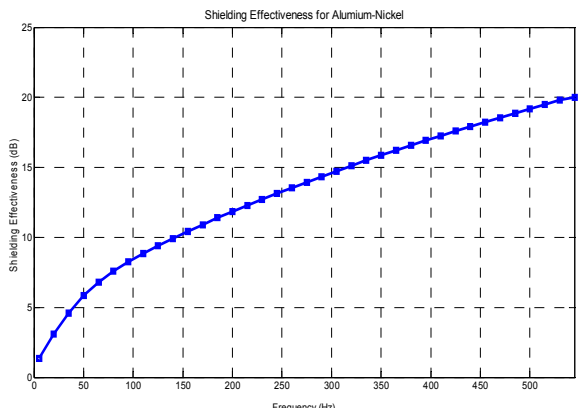

(b)

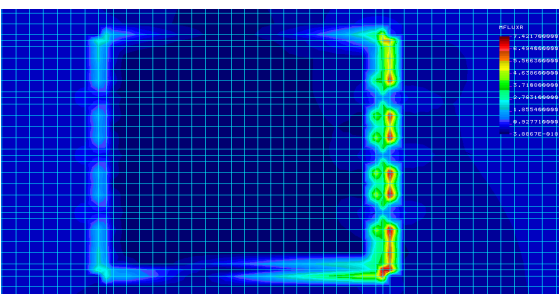

(a)

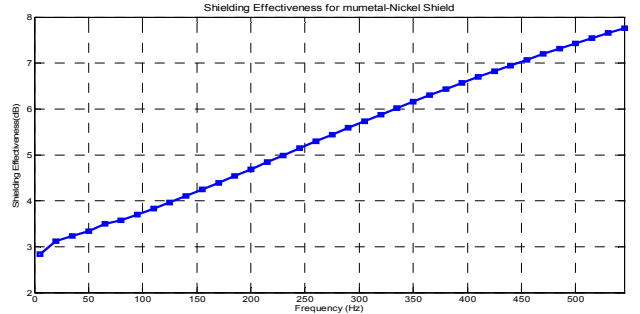

(b)

Fig.25. (a) Contour plot, (b) shielding effectiveness on nickel- mumetal shield

Indeed, in composite shield, the shields influence on together and connection type of them are important factor to cause increase or decrease in shielding effectiveness. Multilayer shields are suitable to safekeeping against interference pulses. Multilayer shields have a lot of application in industry.

\section{CONCLUSION}

Shielding effectiveness is an important parameter to determine, the shield is suitable or unsuitable. In this paper, at first, shielding effectiveness is investigated for high permeable materials, diamagnetic and paramagnetic materials in low frequencies and the effect of shield material is obtained on shield. Then, the effect of magnetic field intensity is investigated on shield's two apertures and six apertures and is obtained the effects of apertures on shielding factor.

If the apertures increase, the shielding effectiveness will decrease and this is undesirable for shield design. This result 
has been shown in simulations. In following, the effect of aperture position displacement is investigated on shielding factor. The aperture position is important factor for determining suitable shielding effectiveness. It can propose to find optimum position's aperture for shield in the next papers. Finally, the effect of composite shield is investigated on shielding effectiveness. The shielding effectiveness is influenced of composite shield severely. The aim of this paper, investigations of some shielding design parameters and influence them on shielding effectiveness.

\section{REFERENCES}

[1] M. Sandstrom,K.H. Mild, M.Sandstrom, and A.Berglund., "External power frequency magnetic field-induced jitter on computer monitors," Behaviour\&Inform.Technol,Vol. 12, No. 6, 359-363, 1993

[2] T. Williams, EMC for Product Designers, Newnes, Great Britain, oxford library, 2001.

[3] Z.Yichao, G.Cheng, S. Lihua, Z.Bihua, "analysis and test EM shielding for low frequency magnetic field" IEEE conference, 345-349, 2007.

[4] S.A. Schelkunoff, "Electromanetic Waves," van Nostrand, Princeton NJ, 1943.

[5] J. F. Hoburg, "principle of quasistatic magnetic shielding with cylindrical and spherical shields," IEEE Trans. Electromagn. Compat., Vol.37, No.4, pp.574-579, Nov.1995.

[6] R.P.Clayton,Introduction to Electromagnetic Compatibility, Wiley-Interscience, New York, 2006.

[7] I. Straus, C.Straus, "Magnetic Field Calibration: Unwinding the Helmholtz Coil,"'LLC Littleton, A, Conformity 2002.

[8] L. Hasselgren, J.Luomi, "Geometrical Aspects of Magnetic Shielding at Extremely High Frequency," IEEE Transactions on Electromagnetic compatibility, Vol. 37, No. 3, 1995.

[9] ETS. LINDGREN, "Helmholtz Coil Manual," EMC Test System, Dec 2001.

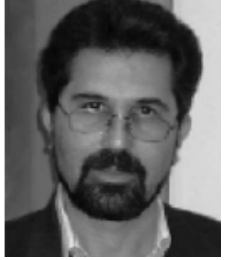

Asghar Keshtkar, was born in Ardabil, Iran, in 1962. $\mathrm{He}$ received the B.Sc. degree in electrical engineering from Tehran University, Tehran, Iran, in 1989, the M.Sc. degree in electrical engineering from the University of Khaje-Nasir, Tehran, in 1992, and the $\mathrm{Ph} . \mathrm{D}$. degree in electrical engineering from the Iran University of Science and Technology, Tehran, in 1999. His research interests include electromagnetic launcher, Electromagnetic Compatibility (EMC),

bioelectromagnetics, and antenna Keshtkar is currently an Associate Professor with the Faculty of Engineering and Technology, Imam Khomeini International University, Ghazvin, Iran.

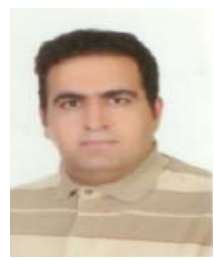

Amir Maghoul, was born in Amlash, Guilan, Iran, in 1983. He received the B.Sc. degree in electrical engineering, Guilan, Iran, in 2005, the M.Sc degree in communication field and wave engineering from the University of Tabriz, Tabriz, Iran, in 2008.

His research interests include Electromagnetic Compatibility (EMC), radar systems design, and antenna design. Mr. Maghoul is currently a researcher with EMC department in space research center of Iran.

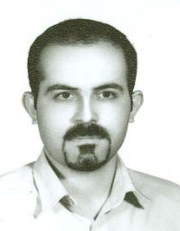

Ali kalantarnia, was born in Hamedan, Iran, in 1980 $\mathrm{He}$ received the B.Sc. degree in electrical engineering from Bu AliSina University, Hamedan, Iran, 2003, the M.Sc. degree in communication field and wave engineering from the University of Tabriz, Tabriz, Iran, 2006.

His research interest includes Electromagnetic Compatibility (EMC), Electromagnetic Launcher and

Optical Communication. Mr. Kalantarnia is currently a Faculty member of Applied and Science Technology, Hamedan, Iran. 